\title{
SPECTROSCOPIC CONFIRMATION OF THE DWARF GALAXIES HYDRA II AND PISCES II AND THE GLOBULAR CLUSTER LAEVENS $1^{*}$
}

\author{
Evan N. Kirby ${ }^{1}$, Joshua D. Simon ${ }^{2}$, and Judith G. Cohen ${ }^{1}$ \\ ${ }^{1}$ California Institute of Technology, 1200 E. California Blvd., MC 249-17, Pasadena, CA 91125, USA \\ ${ }^{2}$ Observatories of the Carnegie Institution of Washington, 813 Santa Barbara Street, Pasadena, CA 91101, USA \\ Received 2015 June 2; accepted 2015 July 28; published 2015 August 28
}

\begin{abstract}
We present Keck/DEIMOS spectroscopy of stars in the recently discovered Milky Way satellites Hydra II, Pisces II, and Laevens 1. We measured a velocity dispersion of $5.4_{-2.4}^{+3.6} \mathrm{~km} \mathrm{~s}^{-1}$ for Pisces II, but we did not resolve the velocity dispersions of Hydra II or Laevens 1. We marginally resolved the metallicity dispersions of Hydra II and Pisces II but not Laevens 1. Furthermore, Hydra II and Pisces II obey the luminosity-metallicity relation for Milky Way dwarf galaxies $(\langle[\mathrm{Fe} / \mathrm{H}]\rangle=-2.02 \pm 0.08$ and $-2.45 \pm 0.07$, respectively), whereas Laevens 1 does not $(\langle[\mathrm{Fe} / \mathrm{H}]\rangle=-1.68 \pm 0.05)$. The kinematic and chemical properties suggest that Hydra II and Pisces II are dwarf galaxies, and Laevens 1 is a globular cluster. We determined that two of the previously observed blue stars near the center of Laevens 1 are not members of the cluster. A third blue star has ambiguous membership. Hydra II has a radial velocity $\left\langle v_{\text {helio }}\right\rangle=303.1 \pm 1.4 \mathrm{~km} \mathrm{~s}^{-1}$, similar to the leading arm of the Magellanic stream. The mass-tolight ratio for Pisces II is $370_{-240}^{+310} M_{\odot} / L_{\odot}$. It is not among the most dark matter-dominated dwarf galaxies, but it is still worthy of inclusion in the search for gamma-rays from dark matter self-annihilation.
\end{abstract}

Key words: galaxies: abundances - galaxies: dwarf - Local Group

\section{INTRODUCTION}

Ultra-faint dwarf galaxies (UFDs) harbor a wealth of information about dark matter and nucleosynthesis. They are the most dark matter-dominated objects known (Simon \& Geha 2007). Their large dark-to-luminous mass ratios and their small sizes imply large central densities of dark matter. Hence, they are excellent targets for the detection of gamma-rays from dark matter self-annihilation (Bonnivard et al. 2015). They also contain the largest concentrations of metal-poor stars of any galaxy type (Kirby et al. 2008b; Frebel et al. 2014). The most metal-poor stars in UFDs could be the direct descendants of the first generation of stars in the universe. If so, then their compositions are direct samples of Population III nucleosynthesis (Frebel et al. 2010).

The Sloan Digital Sky Survey (SDSS; Abazajian et al. 2009) revolutionized the field of dwarf galaxies a decade ago by discovering the first UFDs. In total, more than a dozen new UFDs were found in the SDSS (e.g., Willman et al. 2005; Zucker et al. 2006; Belokurov et al. 2007). In the span of roughly five years, SDSS and its successor, the Sloan Extension for Galactic Understanding and Evolution (SEGUE, Yanny et al. 2009), more than doubled the number of known Milky Way satellites. The field is experiencing another rejuvenation with the arrival of several new imaging surveys with deeper photometry and/or coverage of the southern sky: the Dark Energy Survey (DES), the Survey of the MAgellanic Stellar History (SMASH; Nidever et al. 2015), the Panoramic Survey Telescope and Rapid Response System (Pan-STARRS, Kaiser et al. 2010), ATLAS at the Very Large Telescope (VLT) Survey Telescope (Shanks et al. 2015), and independent imaging with the Dark Energy Camera (DECam) at the CTIO/

\footnotetext{
* The data presented herein were obtained at the W.M. Keck Observatory, which is operated as a scientific partnership among the California Institute of Technology, the University of California and the National Aeronautics and Space Administration. The Observatory was made possible by the generous financial support of the W.M. Keck Foundation.
}

Blanco telescope. To date, DECam imaging, including DES, has enabled the discovery of 12 new Milky Way satellites (Bechtol et al. 2015; Kim \& Jerjen 2015a, 2015b; Kim et al. 2015a, 2015b; Koposov et al. 2015a). Pan-STARRS discovered two satellites (Laevens et al. 2014, 2015), and ATLAS and SMASH have each discovered one (Belokurov et al. 2014; Martin et al. 2015). ${ }^{3}$ Together, these surveys discovered 15 additional satellites in less than a year and a half. Their success can be attributed to deeper photometry and surveying a different part of the sky than SDSS.

The photometric discovery is the first step in identifying a new dwarf galaxy. In order to classify a stellar system as a galaxy, it should exhibit some evidence for dark matter in the form of a large mass-to-light ratio $(M / L)$, metallicity dispersion, or both (Willman \& Strader 2012). These criteria can be tested only with spectroscopy. Two of the newly discovered satellites, Reticulum II and Horologium I, have been spectroscopically confirmed as dwarf galaxies by both velocity and metallicity dispersions (Koposov et al. 2015b; Simon et al. 2015; Walker et al. 2015). The other systems have tentative classifications as galaxies or globular clusters (GCs) based on their luminosities and half-light radii $\left(r_{\mathrm{h}}\right)$. GCs have $r_{\mathrm{h}}<30 \mathrm{pc}$ regardless of luminosity (Harris 1996), but the $r_{\mathrm{h}}$ of galaxies increases with luminosity (e.g., Belokurov et al. 2007). The half-light radii of dwarf galaxies overlap with GCs $\left(r_{\mathrm{h}} \approx 30 \mathrm{pc}\right)$ at $M_{V} \approx-2$, and they grow to $r_{\mathrm{h}}>100 \mathrm{pc}$ at $M_{V}<-5$.

This study concerns three recently discovered satellites. Martin et al. (2015) discovered Hydra II in DECam images taken as part of SMASH. Its large half-light radius, $68 \pm 11 \mathrm{pc}$, strongly suggests that it is a dwarf galaxy. Hydra II is especially interesting for its proximity to the Magellanic Clouds. Martin et al. raised the possibility that it is associated with the LMC, which is potentially true for many of the DESdiscovered satellites (Bechtol et al. 2015; Deason et al. 2015;

\footnotetext{
3 Laevens et al. (2014), using Pan-STARRS, discovered Laevens 1 simultaneously with Belokurov et al. (2014), who used ATLAS.
} 

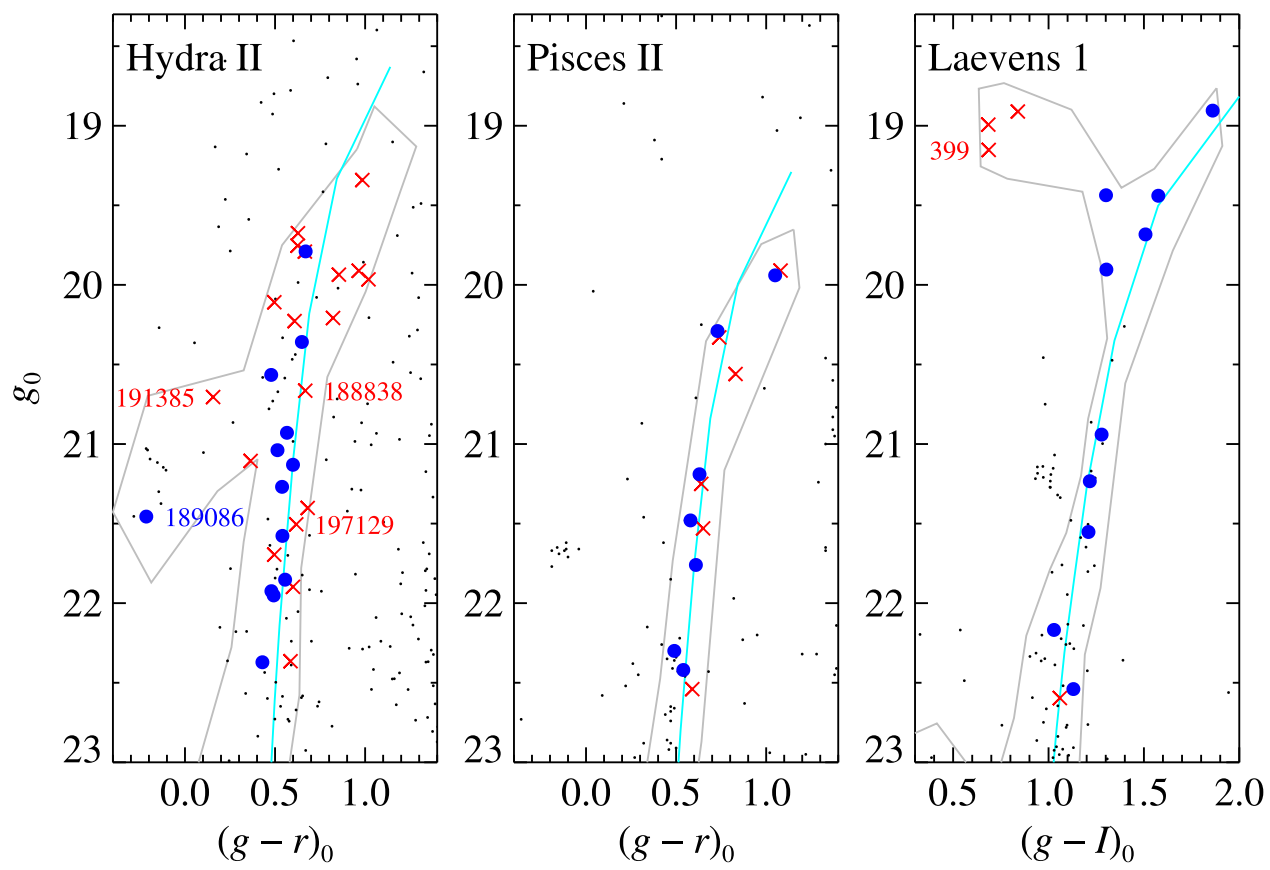

Figure 1. Color-magnitude diagrams of the three satellites. Blue points are spectroscopically confirmed members, whereas red crosses are non-members. Small black points are stars within $4^{\prime}$ of the satellite's center that we did not observe spectroscopically. The gray regions circumscribe the stars that were considered possible members. The ridgeline for the metal-poor GC M92 (Clem 2006) is shown in cyan.

Koposov et al. 2015a). Belokurov et al. (2010) discovered Pisces II in SDSS images, and they confirmed an overdensity of main sequence turn-off stars with deeper KPNO/MOSAIC images. Its size, $r_{\mathrm{h}} \approx 60 \mathrm{pc}$, suggests that it is also a galaxy. However, no spectroscopy has been obtained since its discovery. Belokurov et al. (2014) and Laevens et al. (2014) co-discovered Laevens $1 / \mathrm{Crater}^{4}$ with ATLAS and PanSTARRS, respectively. Its half-light radius is only about $20 \mathrm{pc}$, and its luminosity is $M_{V} \approx-5$. Therefore, it can be tentatively classified as a GC, but spectroscopy is required for a definitive identification.

We observed Hydra II, Pisces II, and Laevens 1 with Keck/ DEIMOS in order to identify them as galaxies or clusters. We completed the identification by measuring both velocity and metallicity dispersions. We also quantified the metallicities of a few stars in these galaxies to get a hint of their capability to enhance their own metallicities.

\section{PHOTOMETRY AND ASTROMETRY}

In order to design DEIMOS slitmasks, we needed a catalog of coordinates for stars that were potentially members of the three satellites. We also needed colors and magnitudes of stars to identify candidate members. Sand et al. (2012) published a photometric and astrometric catalog for Pisces II, but no such published catalog exists for Hydra II or Laevens 1. We downloaded publicly available images for Hydra II, and we obtained new Keck/LRIS images of Laevens 1.

\subsection{Hydra II}

Martin et al. (2015) discovered Hydra II in SMASH/DECam images. The Hydra II images, taken on 2013 March 20, are publicly available through the NOAO Science Archive. ${ }^{5}$ We

\footnotetext{
4 We call the object Laevens 1, as is the convention for GCs.

5 http://www.portal-nvo.noao.edu/
}

downloaded the calibrated images in the DECam $g$ and $r$ filters. These images are flat fielded, and they have astrometry headers. We used SExtractor (Bertin \& Arnouts 1996) to identify stars and measure their magnitudes in each of the $g$ and $r$ images. We discarded objects with class_star $\leqslant 0.8$ in order to weed out galaxies and image artifacts. We corrected magnitudes for extinction according to the dust maps of (Schlegel et al. 1998, SFD98).

We selected stars for spectroscopy based on their colors and magnitudes. We drew a region in the color-magnitude diagram (CMD) in the approximate shape of the red giant branch (RGB) and horizontal branch (HB). Figure 1 shows this region in gray. We assigned priorities for spectroscopic selection within the region based on magnitude but not on color. These priorities were used to resolve conflicts where slitmask design constraints forced a choice among two or more stars. Brighter stars were given higher priorities. The stars that were able to be placed on the DEIMOS slitmask are shown as blue points (members) and red crosses (non-members). Figure 2 shows the sky coordinates of the spectroscopic targets.

Our spectroscopic selection includes two potential HB stars, 189086 and 191385, and one asymptotic giant branch (AGB) star, 194563. Our kinematic membership selection (Section 5.1) includes all three stars. However, star 191385 is 0.4 mag above the HB. Therefore, we ruled it a non-member. Star 189086 has a color and magnitude consistent with the HB. Although it is the most distant member in our Hydra II sample, we kept it in our list of members. These decisions do not affect our results in a measurable way (Section 5.2).

\subsection{Pisces II}

Sand et al. (2012) observed Pisces II with Magellan/ Megacam in the $g$ and $r$ filters. We used their published astrometric and photometric catalog. They corrected for extinction using the SFD98 dust map. We drew a spectroscopic 

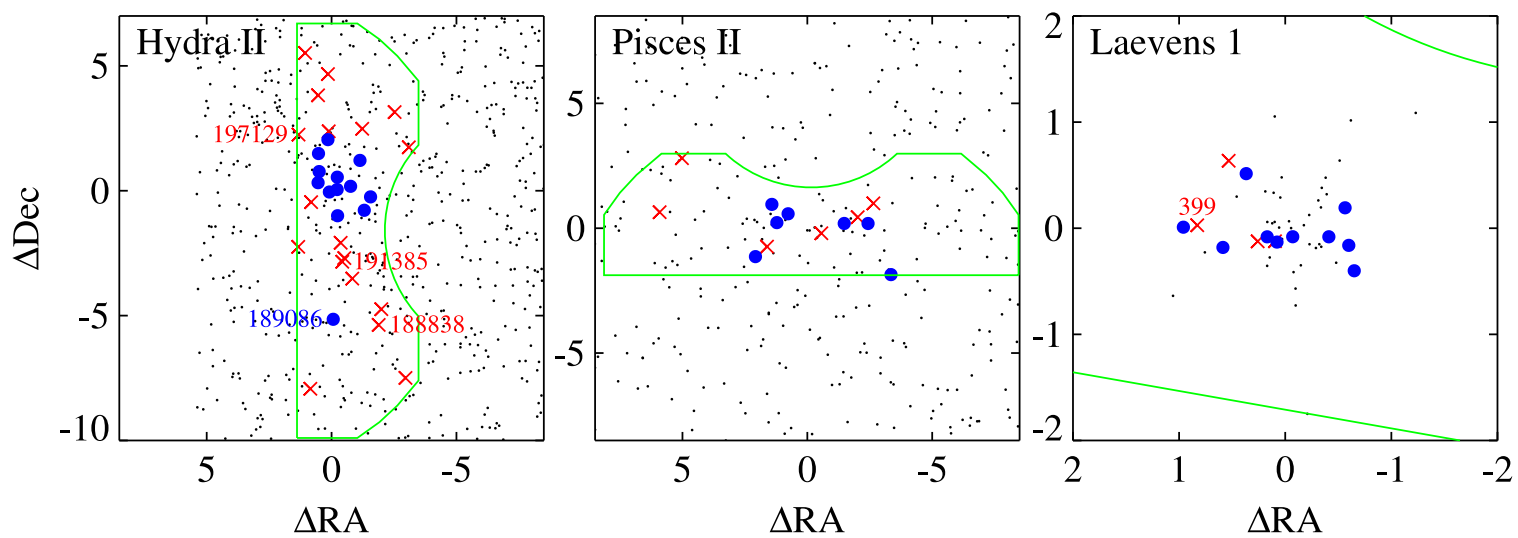

Figure 2. Sky coordinates of spectroscopic targets. The values shown are displacements from the satellite center, as measured by Martin et al. (2015), Belokurov et al. (2010), and Laevens et al. (2014). Blue points are spectroscopically confirmed members, whereas red crosses are non-members. Small black points are stars with $g_{0}<22$ that were not targeted for spectroscopy. The DEIMOS slitmask outline is shown in green. Because the field for Laevens 1 is smaller than the other two satellites, the axis ranges are smaller.

selection region around the RGB (Figure 1). Because Pisces II has a smaller half-light radius (1!1, Sand et al. 2012) than Hydra II (1!7, Martin et al. 2015), the possible targets are denser on the sky, which causes more conflicts for spectroscopic selection. As a result, the selection region did not include the HB so that RGB stars could be selected instead. (RGB spectra lend themselves more easily to the measurements of radial velocity and metallicity than $\mathrm{HB}$ spectra.) As for Hydra II, spectroscopic priority was given to brighter stars.

\subsection{Laevens 1}

D. Perley kindly observed Laevens 1 for us with Keck/LRIS (Oke et al. 1995) on 2015 March 23. Simultaneous exposures were obtained for $130 \mathrm{~s}$ in the $g$ filter in the blue arm and $120 \mathrm{~s}$ in the $I$ filter in the red arm. The images were reduced with LPipe, ${ }^{6}$ which provides flat fielding, astrometric solutions, and photometric calibration.

We identified stars and measured their magnitudes with DAOPHOT (Stetson 1987, 2011). The LRIS point-spread function (PSF) was asymmetric, and it varied over the field. We allowed DAOPHOT to choose the best analytic PSF, which was a Penny function (a two-dimensional Gaussian core with Lorentzian wings). Additionally, there was a look-up table that allowed the PSF to vary linearly over the field. Again, we corrected for extinction using the SFD98 dust map.

Because the half-light radius of Laevens 1 (0.5-0.6, Belokurov et al. 2014; Laevens et al. 2014) is even smaller than Pisces II, we again selected stars on the RGB, not the HB. However, we also included three bright, blue stars near the center of the system. The nature of these stars is controversial. Belokurov et al. (2014) speculated that these are core heliumburning blue loop stars, which would imply that Laevens 1 has formed stars within the last few hundred Myr, making it a dwarf galaxy. Laevens et al. (2014) also identified these stars, but they did not favor their interpretation as blue loop stars because young, blue stars at fainter magnitudes were absent. We obtained spectra of all three stars in order to determine if they are members of Laevens 1 . We also note that Bonifacio et al. (2015) found a population of stars brighter and bluer than the main sequence turn-off but too faint for spectroscopy. If

\footnotetext{
6 http://www.astro.caltech.edu/ dperley/programs/lpipe.html
}

they are not blue stragglers, these stars could signify the presence of a $\sim 2$ Gyr old population in Laevens 1 .

For the purposes of measuring metallicities, it is convenient to have photometry in a uniform system. Therefore, we converted Cousins $I$ magnitudes into SDSS $i$ magnitudes following the conversion formula of Jordi et al. (2006): $i=I+0.21(R-I)+0.34$. The formula depends weakly on $R-I$ color, which we approximated as 0.5 mag for all RGB stars. Due to the imprecision of this assumption, we added 0.05 in quadrature to the $i$ photometric errors. This error floor corresponds to an error in $R-I$ color of $0.2 \mathrm{mag}$. For comparison, the full range of $R-I$ color for an old, metalpoor RGB is about $0.4 \mathrm{mag}$.

\section{SPECTROSCOPY}

\subsection{Observations}

We observed one slitmask for each satellite with DEIMOS (Faber et al. 2003) on 2015 May 18. Table 1 lists the exposure times of each slitmask. We used the $1200 \mathrm{G}$ grating with a ruling of 1200 lines $\mathrm{mm}^{-1}$ and a blaze wavelength of $7760 \AA$. The grating was tilted such that the center of the CCD mosaic corresponded to $7800 \AA$ A. Slits were 0 ." 7 wide. This configuration gives an approximate wavelength range of $6300-9100 \AA$ at a resolution of $1.3 \AA$ FWHM $(R \sim 6000$ at $7800 \AA)$. The exact wavelength range of each spectrum depends on the location of the slit on the slitmask. The starting and ending wavelengths of the spectra vary by $\sim 300 \AA$ across the slitmask. We also obtained internal flat field and arc lamp exposures in the afternoon and morning for calibration.

As mentioned above, Belokurov et al. (2014) identified several bright, blue stars in the vicinity of Laevens 1 . These stars would be unusual in an old GC or dwarf galaxy. Our slitmask included two of these stars. We observed another blue star, 1717 , with a single 0 ". 7 slit.

We also observed nine radial velocity standard stars and two telluric (hot) stars with a long slit. The slit width was 0 ".7. We used a long slit that spanned the entire length of the DEIMOS field of view. This allowed us to refine the wavelength solution based on night sky lines over the entire CCD mosaic. Section 3.2 describes our approach to the wavelength solution. 
Table 1

DEIMOS Observations

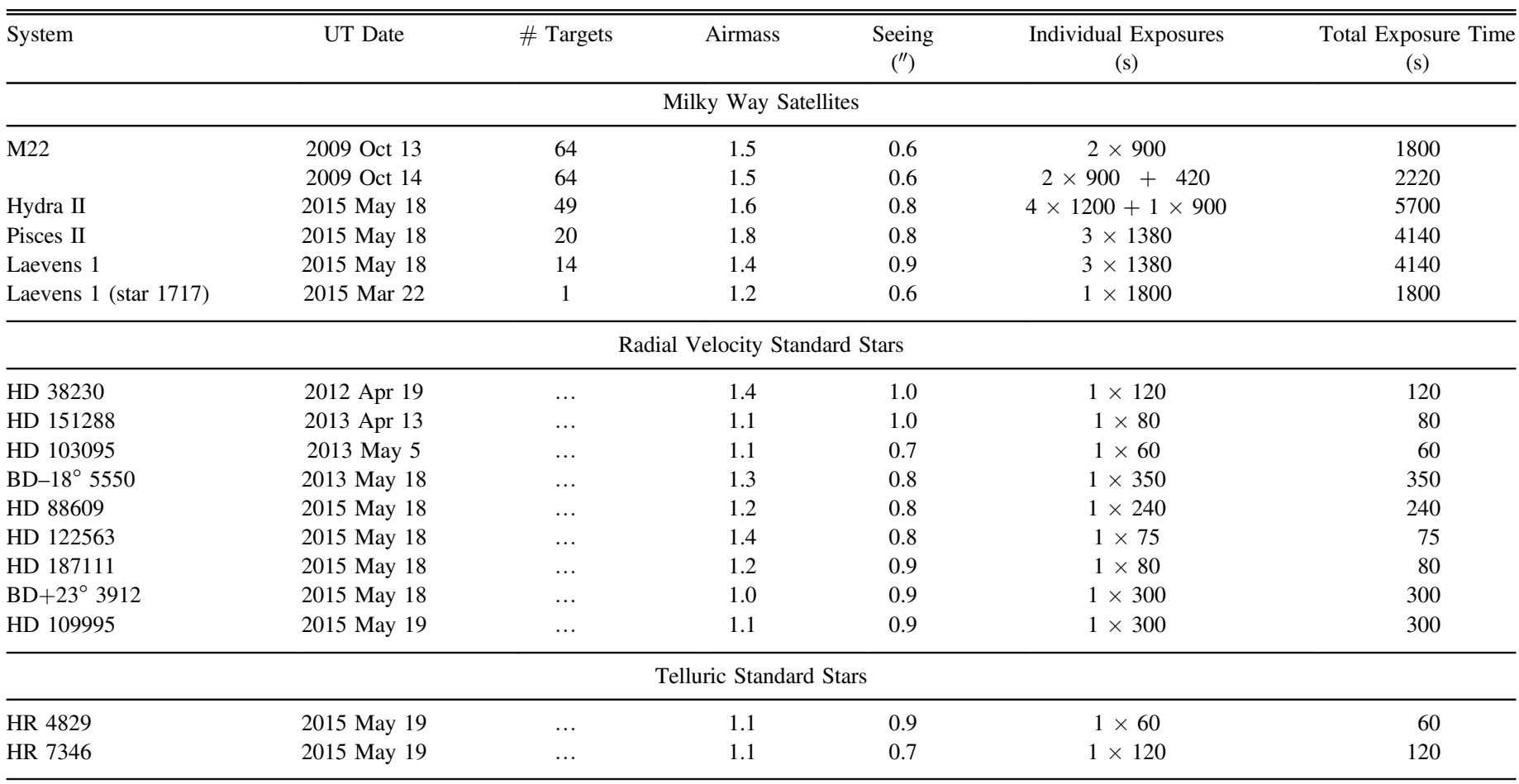

\subsection{Reductions}

As in our previous papers (e.g., Simon \& Geha 2007; Simon et al. 2011; Kirby et al. 2013a, 2015), we reduced the DEIMOS spectra using a slightly modified version of the spec $2 \mathrm{~d}$ IDL data reduction pipeline developed by the DEEP2 team (Cooper et al. 2012; Newman et al. 2013). We introduced a few updates to those procedures for this data set. The updates include improvements to the wavelength solution as determined from sky emission lines and corrections for the effects of differential atmospheric refraction. In the first stage of the sky line wavelength tweaking, a zero point offset is determined during the main reduction pipeline. We improved the tracing of the sky lines across each slit at low signal-to-noise ratio $(\mathrm{S} / \mathrm{N})$, which is particularly important for our long-slit observations of bright template stars with short integration times. We also added a second stage to the sky line fitting: after the reductions are completed, a quadratic fit is performed to the sky line wavelengths in each extracted spectrum, and then the variation of each of the quadratic fit parameters as a function of position on the slit mask is fit with a polynomial. This process corrects for errors in the flexure compensation system as well as temperature changes between the times at which the arc frames and the science frames were obtained (M. Geha et al. 2015, in preparation).

Atmospheric refraction shifts the position of the star light within the slit during the observations. Shifts in the spatial direction (along the slit) result in curvature of the object profile on the detector, and we changed the DEEP2 extraction algorithm to account for this effect (Kirby et al. 2015). Shifts in the wavelength direction (across the slit) result in velocity offsets that vary as a function of wavelength. Using the airmass at the time of observation, the angle between the slit and the parallactic angle, and the measured seeing, we computed a correction for this velocity shift and applied it to the extracted spectra.

In order to take maximum advantage of these improvements, we constructed a new empirical library of template stars for radial velocity measurements to replace the template set most DEIMOS dwarf galaxy studies have employed since Simon \& Geha (2007). We selected a sample of metal-poor stars spanning a range of effective temperature, surface gravity, and metallicity, and we observed them by orienting the slit north-south and slowly driving the telescope such that the star moved steadily across the slit. This process ensured that the star light uniformly illuminated the slit during the exposure. To make sure that the template star observations contained strong enough sky lines for accurate adjustments to the wavelength solution (as described above), we integrated for at least $60 \mathrm{~s}$ during each template observation even if the star crossed the slit in a much shorter amount of time. We also replaced the telluric template from Simon \& Geha (2007) with higher S/N observations using the same techniques.

Figure 3 shows example 1D spectra of one member star in each satellite. The spectra shown for Hydra II and Pisces II have high $\mathrm{S} / \mathrm{N}$, while the spectrum for Laevens 1 has low $\mathrm{S} / \mathrm{N}$. Also shown are the best-fit model spectra (described in Section 4.2) for the stars with $\mathrm{S} / \mathrm{N}$ high enough to measure metallicity. Because the $\mathrm{S} / \mathrm{N}$ is low for the star in Laevens 1 , we did not measure a metallicity for it, and no model spectrum is shown.

\section{SPECTROSCOPIC MEASUREMENTS}

\subsection{Radial Velocity Measurements}

We measured radial velocities of stars in the three Milky Way satellites by comparing their spectra to the radial velocity template stars' spectra. In a manner similar to Simon \& Geha 


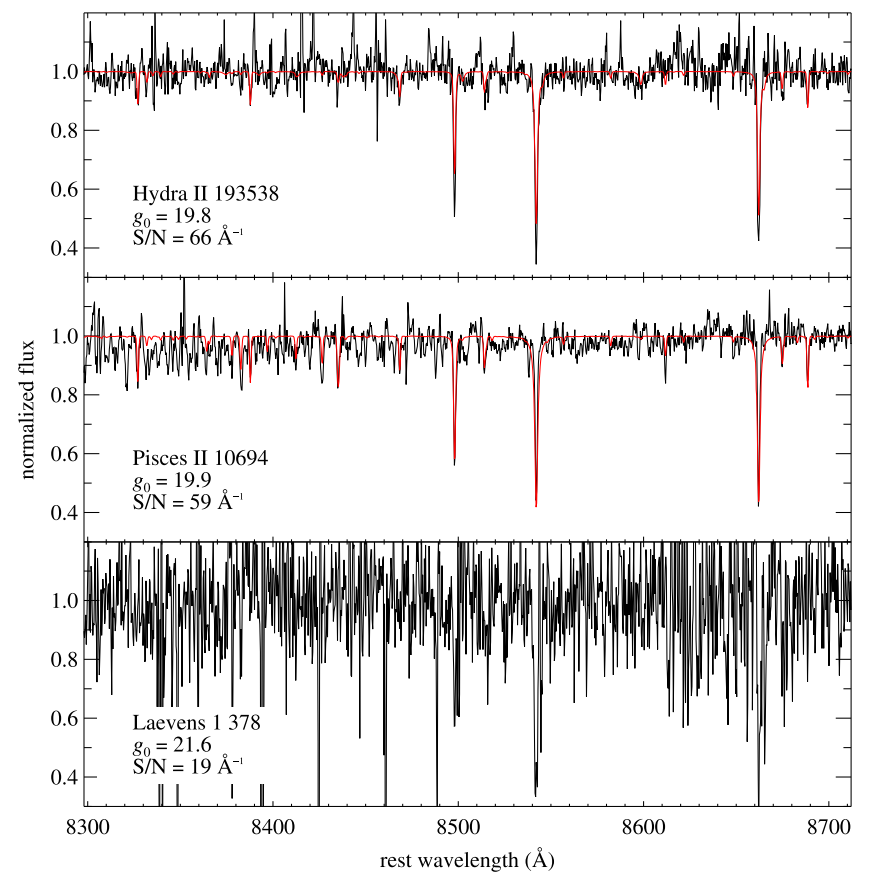

Figure 3. Example spectra for one red giant in each of the satellites. The top two spectra have high $\mathrm{S} / \mathrm{N}$, and the bottom spectrum has low $\mathrm{S} / \mathrm{N}$. We fit model spectra (red) to the high-S/N spectra (Section 4.2). Pisces II 10694 is carbon-rich with $\mathrm{CN}$ absorption visible between 8300 and $8400 \AA$. The model spectrum has a normal carbon abundance, so there is excess $\mathrm{CN}$ absorption in the observed spectrum compared to the model.

(2007), we optimized the DEEP2 survey's redshift measurement technique (Newman et al. 2013) for measuring stellar velocities rather than galaxy redshifts. This technique involves computing $\quad \chi^{2}=(\text { target flux }- \text { template flux })^{2} /(\text { flux error })^{2}$ for each template star. The relative velocity between the target and the template is shifted to find the minimum $\chi^{2}$. The velocity of the star $\left(v_{\text {obs }}\right)$ is the one that minimizes $\chi^{2}$ among all of the templates. As Newman et al. pointed out, this technique is a generalization of a cross-correlation (Tonry \& Davis 1979) that allows for different values of flux error for each pixel.

Small astrometric errors or imprecision in the alignment of the slitmask cause each star to fall at some displacement from the exact center of the slit. Mis-centering translates to a shift in the wavelength scale. Following Simon \& Geha (2007), we corrected all of the stellar velocities to a standard geocentric frame by finding the velocity offset $\left(v_{\text {geo }}\right)$ required to align the observed telluric absorption features with the features in the hot star spectra. The observed wavelength regions included in the

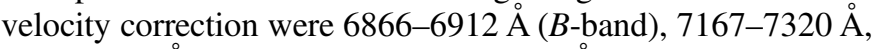
$7593-7690 \AA$ ( $A$-band), and $8110-8320 \AA$. The velocity corrections were determined with the $\chi^{2}$ technique described in the previous paragraph. The velocities quoted in this paper are in the heliocentric frame: $v_{\text {helio }}=v_{\text {obs }}+v_{\text {geo }}+v_{\text {corr }}$, where $v_{\text {corr }}$ is the conversion from the geocentric frame to the heliocentric frame.

We estimated random and systematic uncertainties separately. To estimate the random uncertainty, we resampled the spectra for $10^{3}$ Monte Carlo trials. In one trial, the flux of each pixel was drawn from a Gaussian probability distribution. The mean of the distribution was the original flux value, and its variance was the flux variance estimated by spec $2 \mathrm{~d}$. We remeasured $v_{\text {helio }}$ (including both $v_{\text {obs }}$ and $v_{\text {geo }}$ ) for all of the trials, and we took the random uncertainty, $\delta_{\text {rand }} v$, to be the standard deviation of the $v_{\text {helio }}$ measurements.

There is some minimum velocity uncertainty, even for noiseless spectra. This uncertainty can arise due to uncorrected flexure in DEIMOS, spectral template mismatches, errors in the wavelength solution, and unknown causes. We estimated $\delta_{\text {sys }} v$ by calculating the difference in $v_{\text {helio }}$ for two independent measurements of the same set of stars. The ideal sample for estimating $\delta_{\text {sys }} v$ will have many high-S $/ \mathrm{N}$ spectra. Instead of using the dwarf galaxy sample, we used spectra of 52 red giants in the GC M22. The spectra were taken with the same slitmask on consecutive nights (see Table 1). The data from each night were reduced separately according to Section 3.2. We measured $v_{\text {helio }}$ and estimated $\delta_{\text {rand }} v$ as described above. We then calculated the quantity $\left(v_{\text {helio,1 }}-v_{\text {helio,2 }}\right) /$ $\sqrt{\delta_{\text {rand, } 1} v^{2}+\delta_{\text {rand }, 2} v^{2}+2 \delta_{\text {sys }} v^{2}}$ for each pair of observations of the same star. The standard deviation of this quantity should be 1 for well-determined errors. The value of $\delta_{\text {sys }} v$ required to satisfy that condition is $1.49 \mathrm{~km} \mathrm{~s}^{-1}$. The final error bar for each star is $\sqrt{\delta_{\text {rand }} v^{2}+\delta_{\text {sys }} v^{2}}$.

Our estimate of $\delta_{\text {sys }} v$ ignores some potentially important sources of error. First, the repeat measurements were obtained with the same slitmask. As a result, some of the slit miscentering-for example, due to astrometric errors in the slitmask design — would be repeated in the same way across the two consecutive nights. Second, the slitmasks were observed at about the same hour angle on each night. Although we applied wavelength corrections for flexure and differential atmospheric refraction, these errors would have been of the same sign and similar magnitude for both nights. Any residual, uncorrected error would be similar across both nights. Third, the M22 spectra have fairly high S/N. Consequently, the same template spectrum was assigned to 47 out of 52 M22 stars. This fraction would be lower in spectra with lower $\mathrm{S} / \mathrm{N}$.

All of these effects would potentially lower the measurement of $\delta_{\text {sys }} v$ artificially. In order to quantify the effect of a spuriously low value of $\delta_{\text {sys }} v$, we also calculated velocity dispersions as described in Section 5.2, assuming $\delta_{\text {sys }} v=2.2$ $\mathrm{km} \mathrm{s}^{-1}$, the original value calculated by Simon \& Geha (2007), instead of $1.49 \mathrm{~km} \mathrm{~s}^{-1}$. The increased systematic error would make the upper limits on the velocity dispersions about $5 \%$ less stringent, and it would reduce the measured velocity dispersion of Pisces II by $13 \%$, well within the uncertainty. In summary, our lower value of $\delta_{\text {sys }} v$ than previous works does not change our conclusions.

Table 2 presents the velocities for stars in the satellite galaxies. The table excludes stars where velocity errors exceeded $30 \mathrm{~km} \mathrm{~s}^{-1}$ and stars where velocity measurement was not possible. Figure 4 shows velocity histograms for each satellite in bins of $2.5 \mathrm{~km} \mathrm{~s}^{-1}$. The domain of the plots is $100 \mathrm{~km} \mathrm{~s}^{-1}$ centered on the mean velocity for each satellite. In Section 5, we use these velocities to measure the kinematic properties of each satellite.

\subsection{Metallicity Measurements}

We measured metallicities by fitting synthetic spectra to the observed spectra following the same procedure as Kirby et al. (2008a, 2010). First, we normalized each spectrum to its continuum. To do so, we divided the spectrum by a spline fit to spectral regions generally free of absorption lines. Then, we matched the spectrum to a grid of synthetic spectra generated 
Table 2

Target List

\begin{tabular}{|c|c|c|c|c|c|c|c|c|c|c|c|}
\hline ID & R. A. (J2000) & decl. (J2000) & $\begin{array}{c}g_{0} \\
(\mathrm{mag})\end{array}$ & $\begin{array}{c}(g-r)_{0} \\
(\mathrm{mag})\end{array}$ & $\begin{array}{l}(g-I)_{0} \\
(\mathrm{mag})\end{array}$ & $\begin{array}{l}\mathrm{S} / \mathrm{N}^{\mathrm{a}} \\
\left(\AA^{-1}\right)\end{array}$ & $\begin{array}{c}\begin{array}{c}v_{\text {helio }} \\
\left(\mathrm{km} \mathrm{s}^{-1}\right)\end{array}\end{array}$ & Member? & $\begin{array}{l}T_{\text {eff }} \\
(\mathrm{K})\end{array}$ & $\begin{array}{c}\log g \\
\left(\mathrm{~cm} \mathrm{~s}^{-2}\right)\end{array}$ & {$[\mathrm{Fe} / \mathrm{H}]$} \\
\hline \multicolumn{12}{|c|}{ Hydra II } \\
\hline 196246 & 122127.50 & -315722.6 & 19.68 & 0.63 & $\ldots$ & 54 & $161.8 \pm 1.6$ & $\mathrm{~N}$ & $\ldots$ & $\ldots$ & $\ldots$ \\
\hline 184647 & 122128.13 & -320636.7 & 19.75 & 0.63 & $\ldots$ & 46 & $116.3 \pm 1.9$ & $\mathrm{~N}$ & $\ldots$ & $\ldots$ & $\ldots$ \\
\hline 199452 & 122130.18 & -315558.0 & 19.94 & 0.85 & $\ldots$ & 59 & $5.1 \pm 1.6$ & $\mathrm{~N}$ & $\ldots$ & $\ldots$ & $\ldots$ \\
\hline 189524 & 122132.70 & -320351.5 & 21.90 & 0.60 & $\ldots$ & 8 & $272.9 \pm 7.9$ & $\mathrm{~N}$ & $\ldots$ & $\ldots$ & $\ldots$ \\
\hline 188838 & 122133.19 & -320429.4 & 20.66 & 0.67 & $\ldots$ & 33 & $319.0 \pm 2.3$ & $\mathrm{~N}$ & $\ldots$ & $\ldots$ & $\ldots$ \\
\hline 194103 & 122134.74 & -315921.8 & 20.93 & 0.57 & $\ldots$ & 25 & $300.0 \pm 4.2$ & $\mathrm{Y}$ & 5095 & 2.05 & $-2.40 \pm 0.32$ \\
\hline 193538 & 122135.93 & -315954.0 & 19.79 & 0.67 & $\ldots$ & 66 & $304.2 \pm 1.7$ & $\mathrm{Y}$ & 4918 & 1.46 & $-1.95 \pm 0.12$ \\
\hline 198021 & 122136.31 & -315638.1 & 21.40 & 0.68 & $\ldots$ & 19 & $63.9 \pm 3.4$ & $\mathrm{~N}$ & $\ldots$ & $\ldots$ & $\ldots$ \\
\hline 195726 & 122136.74 & -315754.1 & 21.13 & 0.60 & $\ldots$ & 22 & $306.5 \pm 3.5$ & $\mathrm{Y}$ & 5036 & 2.11 & $-2.48 \pm 0.33$ \\
\hline 190646 & 122138.18 & -320238.2 & 20.23 & 0.61 & $\ldots$ & 44 & $86.6 \pm 1.8$ & $\mathrm{~N}$ & $\ldots$ & $\ldots$ & $\ldots$ \\
\hline 194563 & 122138.51 & -315856.4 & 20.57 & 0.48 & $\ldots$ & 34 & $302.9 \pm 3.3$ & $\mathrm{Y}$ & $\ldots$ & $\ldots$ & $\ldots$ \\
\hline 191521 & 122139.68 & -320148.8 & 19.91 & 0.96 & $\ldots$ & 45 & $202.5 \pm 1.8$ & $\mathrm{~N}$ & $\ldots$ & $\ldots$ & $\ldots$ \\
\hline $191385^{\mathrm{b}}$ & 122140.08 & -320157.7 & 20.70 & 0.16 & $\ldots$ & 9 & $316.9 \pm 19.9$ & $\mathrm{~N}$ & $\ldots$ & $\ldots$ & $\ldots$ \\
\hline 192206 & 122140.37 & -320112.1 & 19.97 & 1.02 & $\ldots$ & 68 & $28.0 \pm 1.5$ & $\mathrm{~N}$ & $\ldots$ & $\ldots$ & $\ldots$ \\
\hline 193286 & 122140.96 & -320007.0 & 21.27 & 0.54 & $\ldots$ & 14 & $299.9 \pm 5.4$ & $\mathrm{Y}$ & $\ldots$ & $\ldots$ & $\ldots$ \\
\hline 194920 & 122141.02 & -315834.4 & 21.58 & 0.54 & $\ldots$ & 16 & $294.5 \pm 5.9$ & $\mathrm{Y}$ & $\ldots$ & $\ldots$ & $\ldots$ \\
\hline 194405 & 122141.05 & -315904.1 & 20.36 & 0.65 & $\ldots$ & 48 & $304.2 \pm 1.9$ & $\mathrm{Y}$ & 4921 & 1.73 & $-1.89 \pm 0.13$ \\
\hline 189086 & 122141.79 & -320415.9 & 21.46 & -0.21 & $\ldots$ & 4 & $315.5 \pm 23.5$ & $\mathrm{Y}$ & $\ldots$ & $\ldots$ & $\ldots$ \\
\hline 194325 & 122142.51 & -315910.0 & 21.04 & 0.51 & $\ldots$ & 24 & $301.9 \pm 3.0$ & $\mathrm{Y}$ & 5171 & 2.15 & $-2.76 \pm 0.43$ \\
\hline 197616 & 122142.62 & -315644.2 & 21.11 & 0.36 & $\ldots$ & 15 & $94.9 \pm 6.6$ & $\mathrm{~N}$ & $\ldots$ & $\ldots$ & $\ldots$ \\
\hline 201098 & 122142.78 & -315426.7 & 20.21 & 0.82 & $\ldots$ & 22 & $-85.0 \pm 2.3$ & $\mathrm{~N}$ & $\ldots$ & $\ldots$ & $\ldots$ \\
\hline 196797 & 122142.79 & -315704.2 & 22.37 & 0.43 & $\ldots$ & 6 & $300.1 \pm 21.8$ & $\mathrm{Y}$ & $\ldots$ & $\ldots$ & $\ldots$ \\
\hline 195247 & 122144.45 & -315821.0 & 21.92 & 0.48 & $\ldots$ & 11 & $317.5 \pm 16.8$ & $\mathrm{Y}$ & $\ldots$ & $\ldots$ & $\ldots$ \\
\hline 196052 & 122144.57 & -315737.6 & 21.85 & 0.56 & $\ldots$ & 12 & $303.7 \pm 4.1$ & $\mathrm{Y}$ & $\ldots$ & $\ldots$ & $\ldots$ \\
\hline 200162 & 122144.65 & -315517.7 & 19.34 & 0.98 & $\ldots$ & 79 & $68.1 \pm 1.5$ & $\mathrm{~N}$ & $\ldots$ & $\ldots$ & $\ldots$ \\
\hline 194736 & 122144.65 & -315847.6 & 21.95 & 0.49 & $\ldots$ & 10 & $288.3 \pm 14.5$ & $\mathrm{Y}$ & $\ldots$ & $\ldots$ & $\ldots$ \\
\hline 193869 & 122145.96 & -315934.4 & 20.11 & 0.50 & $\ldots$ & 46 & $237.3 \pm 2.1$ & $\mathrm{~N}$ & $\ldots$ & $\ldots$ & $\ldots$ \\
\hline 183842 & 122146.10 & -320702.8 & 19.79 & 0.67 & $\ldots$ & 55 & $-19.6 \pm 1.7$ & $\mathrm{~N}$ & $\ldots$ & $\ldots$ & $\ldots$ \\
\hline 202029 & 122147.09 & -315336.0 & 21.69 & 0.50 & $\ldots$ & 6 & $10.1 \pm 9.0$ & $\mathrm{~N}$ & $\ldots$ & $\ldots$ & $\ldots$ \\
\hline 197129 & 122148.34 & -315652.2 & 21.50 & 0.62 & $\ldots$ & 17 & $315.7 \pm 3.7$ & $\mathrm{~N}$ & $\ldots$ & $\ldots$ & $\ldots$ \\
\hline 192059 & 122148.39 & -320121.8 & 22.36 & 0.59 & $\ldots$ & 7 & $172.4 \pm 10.0$ & $\mathrm{~N}$ & $\ldots$ & $\ldots$ & $\cdots$ \\
\hline \multicolumn{12}{|c|}{ Pisces II } \\
\hline 9004 & 225817.52 & +055517.5 & 20.29 & 0.73 & $\ldots$ & 58 & $-224.9 \pm 1.6$ & $\mathrm{Y}$ & 4787 & 1.34 & $-2.38 \pm 0.13$ \\
\hline 9618 & 225820.35 & +055808.8 & 22.54 & 0.59 & $\ldots$ & 8 & $-301.6 \pm 6.5$ & $\mathrm{~N}$ & $\ldots$ & $\ldots$ & $\ldots$ \\
\hline 9833 & 225821.22 & +055720.3 & 21.76 & 0.61 & $\ldots$ & 19 & $-226.9 \pm 3.2$ & $\mathrm{Y}$ & $\ldots$ & $\ldots$ & $\ldots$ \\
\hline 10215 & 225822.88 & +055735.5 & 21.53 & 0.65 & $\ldots$ & 21 & $-25.9 \pm 3.1$ & $\mathrm{~N}$ & $\ldots$ & $\ldots$ & $\ldots$ \\
\hline 10694 & 225825.06 & +055720.4 & 19.94 & 1.05 & $\ldots$ & 59 & $-232.0 \pm 1.6$ & $\mathrm{Y}$ & 4132 & 0.81 & $-2.70 \pm 0.11$ \\
\hline 11592 & 225828.74 & +055656.9 & 19.91 & 1.08 & $\ldots$ & 82 & $4.3 \pm 1.5$ & $\mathrm{~N}$ & $\ldots$ & $\ldots$ & $\ldots$ \\
\hline 12924 & 225834.10 & +055743.6 & 21.19 & 0.63 & $\ldots$ & 31 & $-221.6 \pm 2.9$ & $\mathrm{Y}$ & 4955 & 1.82 & $-2.10 \pm 0.18$ \\
\hline 13387 & 225835.91 & +055722.4 & 22.30 & 0.49 & $\ldots$ & 11 & $-215.8 \pm 7.6$ & $\mathrm{Y}$ & $\ldots$ & $\ldots$ & $\ldots$ \\
\hline 13560 & 225836.71 & +055806.2 & 21.48 & 0.58 & $\ldots$ & 23 & $-232.6 \pm 5.3$ & $\mathrm{Y}$ & 5049 & 1.99 & $-2.15 \pm 0.28$ \\
\hline 13757 & 225837.49 & +055624.8 & 21.25 & 0.64 & $\ldots$ & 27 & $-102.1 \pm 2.3$ & $\mathrm{~N}$ & $\ldots$ & $\ldots$ & $\ldots$ \\
\hline 14179 & 225839.36 & +055600.7 & 22.42 & 0.54 & $\ldots$ & 8 & $-224.8 \pm 9.6$ & $\mathrm{Y}$ & $\ldots$ & $\ldots$ & $\ldots$ \\
\hline 16716 & 225851.19 & +055957.1 & 20.33 & 0.74 & $\ldots$ & 25 & $-5.1 \pm 10.9$ & $\mathrm{~N}$ & $\ldots$ & $\ldots$ & $\ldots$ \\
\hline 17500 & 225854.78 & +055747.5 & 20.56 & 0.83 & $\ldots$ & 25 & $83.7 \pm 2.2$ & $\mathrm{~N}$ & $\ldots$ & $\ldots$ & $\ldots$ \\
\hline \multicolumn{12}{|c|}{ Laevens 1} \\
\hline 302 & 113613.55 & -105302.9 & 20.94 & $\ldots$ & 1.29 & 20 & $149.8 \pm 2.9$ & $\mathrm{Y}$ & 4977 & 1.53 & $-1.59 \pm 0.15$ \\
\hline 374 & 113613.76 & -105248.5 & 19.90 & $\ldots$ & 1.32 & 68 & $151.9 \pm 1.7$ & $\mathrm{Y}$ & 4966 & 1.09 & $-1.66 \pm 0.11$ \\
\hline 420 & 113613.90 & -105227.3 & 19.44 & $\ldots$ & 1.59 & 105 & $149.8 \pm 1.5$ & $\mathrm{Y}$ & 4626 & 0.69 & $-1.55 \pm 0.11$ \\
\hline 378 & 113614.53 & -105243.7 & 21.55 & $\ldots$ & 1.22 & 19 & $153.5 \pm 3.1$ & $\mathrm{Y}$ & $\ldots$ & $\ldots$ & $\ldots$ \\
\hline 93 & 113615.91 & -105243.6 & 19.68 & $\ldots$ & 1.52 & 88 & $147.2 \pm 1.6$ & $\mathrm{Y}$ & 4726 & 0.84 & $-1.65 \pm 0.11$ \\
\hline 1710 & 113616.52 & -105246.7 & 19.44 & $\ldots$ & 1.31 & 16 & $143.6 \pm 4.5$ & $\mathrm{Y}$ & $\ldots$ & $\ldots$ & $\ldots$ \\
\hline 1715 & 113616.59 & -105246.2 & 18.91 & $\ldots$ & 0.85 & 66 & $72.0 \pm 1.8$ & $\mathrm{~N}$ & $\ldots$ & $\ldots$ & $\ldots$ \\
\hline 367 & 113616.89 & -105243.7 & 22.54 & $\ldots$ & 1.14 & 8 & $153.8 \pm 10.0$ & $\mathrm{Y}$ & $\ldots$ & $\ldots$ & $\ldots$ \\
\hline 1717 & 113617.26 & -105246.2 & 18.99 & $\ldots$ & 0.70 & 47 & $266.0 \pm 2.2$ & $\mathrm{~N}$ & $\ldots$ & $\ldots$ & $\ldots$ \\
\hline 1972 & 113617.70 & -105208.0 & 22.17 & $\ldots$ & 1.04 & 10 & $141.4 \pm 4.7$ & $\mathrm{Y}$ & $\ldots$ & $\ldots$ & $\ldots$ \\
\hline 1997 & 113618.37 & -105200.7 & 22.60 & $\ldots$ & 1.07 & 6 & $166.6 \pm 11.5$ & $\mathrm{~N}$ & $\ldots$ & $\ldots$ & $\ldots$ \\
\hline 1684 & 113618.59 & -105249.7 & 21.23 & $\ldots$ & 1.23 & 21 & $150.9 \pm 3.1$ & $\mathrm{Y}$ & 5064 & 1.70 & $-2.10 \pm 0.21$ \\
\hline 399 & 113619.58 & -105237.1 & 19.15 & $\ldots$ & 0.70 & 80 & $155.3 \pm 1.8$ & $\mathrm{~N}$ & $\ldots$ & $\ldots$ & $\ldots$ \\
\hline
\end{tabular}


Table 2

(Continued)

\begin{tabular}{|c|c|c|c|c|c|c|c|c|c|c|c|}
\hline ID & R. A. (J2000) & decl. (J2000) & $\begin{array}{c}g_{0} \\
(\mathrm{mag})\end{array}$ & $\begin{array}{c}(g-r)_{0} \\
(\mathrm{mag})\end{array}$ & $\begin{array}{c}(g-I)_{0} \\
(\mathrm{mag})\end{array}$ & $\begin{array}{l}\mathrm{S} / \mathrm{N}^{\mathrm{a}} \\
\left(\mathrm{A}^{-1}\right)\end{array}$ & $\begin{array}{c}v_{\text {helio }} \\
\left(\mathrm{km} \mathrm{s}^{-1}\right)\end{array}$ & Member? & $\begin{array}{l}T_{\text {eff }} \\
(\mathrm{K})\end{array}$ & $\begin{array}{c}\log g \\
\left(\mathrm{~cm} \mathrm{~s}^{-2}\right)\end{array}$ & {$[\mathrm{Fe} / \mathrm{H}]$} \\
\hline 963 & 113620.11 & -105238.2 & 18.90 & $\ldots$ & 1.87 & 150 & $149.2 \pm 1.5$ & $\mathrm{Y}$ & 4331 & 0.23 & $-1.78 \pm 0.11$ \\
\hline
\end{tabular}

Notes.

${ }^{\mathrm{a}}$ To convert to $\mathrm{S} / \mathrm{N}$ per pixel, multiply by 0.57 .

${ }^{\mathrm{b}}$ Non-member based on CMD position.
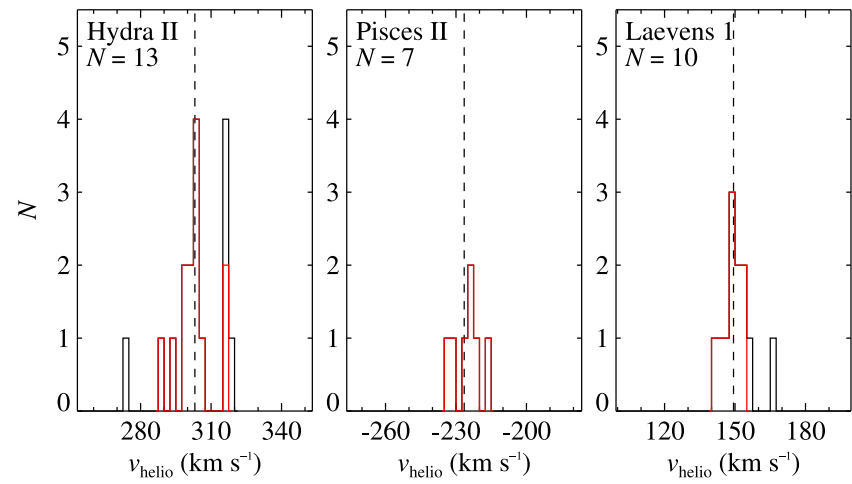

Figure 4. Distributions of radial velocities for stars observed in the field of each satellite. Stars in the red portion of the histograms are deemed members. The dashed line shows $\left\langle v_{\text {helio }}\right\rangle$, and $N$ is the number of member stars. The membership cut includes $99 \%$ of member stars $\left(2.58 \sigma_{v}\right.$ from the mean velocity). Stars whose velocity error bars overlap any part of the allowed velocity range are considered members.

with ATLAS9 model atmospheres (Kurucz 1993) and the synthesis code MOOG (Sneden 1973). We measured iron abundances, $[\mathrm{Fe} / \mathrm{H}],{ }^{7}$ by fitting only to iron absorption lines and ignoring other spectral regions. Please refer to Kirby et al. (2010) for more details.

We estimated the random uncertainty on $[\mathrm{Fe} / \mathrm{H}]$ from the covariance matrix of the $\chi^{2}$ fit to the synthetic spectral grid. There is also a systematic uncertainty of 0.11 dex (Kirby et al. 2010). The final error bar is the quadrature sum of the random and systematic uncertainties.

Table 2 gives effective temperatures $\left(T_{\text {eff }}\right)$, surface gravities $(\log g)$, and $[\mathrm{Fe} / \mathrm{H}]$ measurements for member stars with $\delta[\mathrm{Fe} / \mathrm{H}]<0.5$ and $\mathrm{S} / \mathrm{N}>20 \AA^{-1}$. Figure 5 shows the distribution of these measurements in bins of 0.25 dex. By nature, ultra-faint satellites have only handfuls of red giants bright enough for metallicity measurements. As a result, the metallicity distributions in Figure 5 have only 4-6 stars. Accurate quantification of metallicity distributions and chemical evolution requires larger samples. Nonetheless, we present rudimentary metallicity averages and dispersions in Section 6.

\subsection{Comparison to Other Measurements}

Bonifacio et al. (2015) obtained VLT/X-Shooter spectra of two stars in Laevens 1. Both stars are also in our spectroscopic sample. We call them 420 and 93, and Bonifacio et al. call them J113613-105227 and J113615-105244. They measured a velocity difference between the two stars of $10.2 \pm 5.7 \mathrm{~km} \mathrm{~s}^{-1}$, whereas we measured a difference of only $2.6 \pm 2.2 \mathrm{~km} \mathrm{~s}^{-1}$.

\footnotetext{
7 We adopted the solar abundances of Anders \& Grevesse (1989) except for iron: $12+\log (n(\mathrm{Fe}) / n(\mathrm{H}))=7.52$ (Sneden et al. 1992).
}

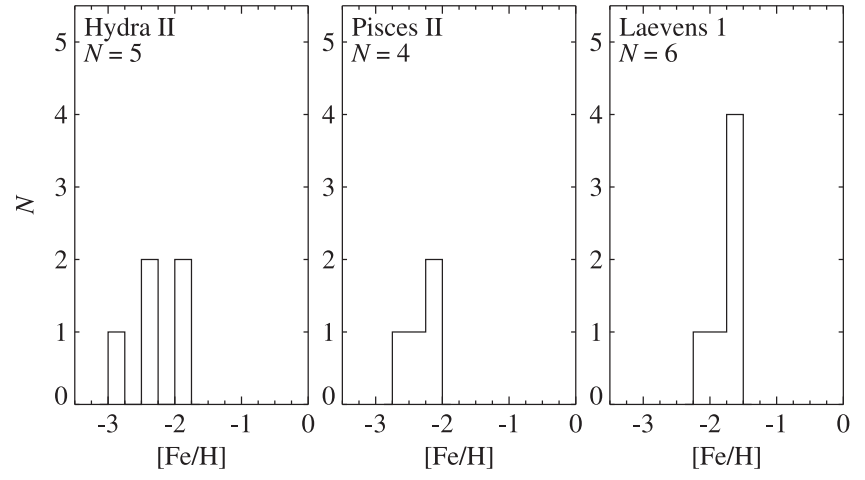

Figure 5. Distribution of $[\mathrm{Fe} / \mathrm{H}]$ in each satellite. There are fewer stars in this figure than in Figure 4 because we could not measure $[\mathrm{Fe} / \mathrm{H}]$ for all radial velocity members. $N$ is the number of stars for which $[\mathrm{Fe} / \mathrm{H}]$ measurements with errors less than $0.5 \mathrm{dex}$ were possible.

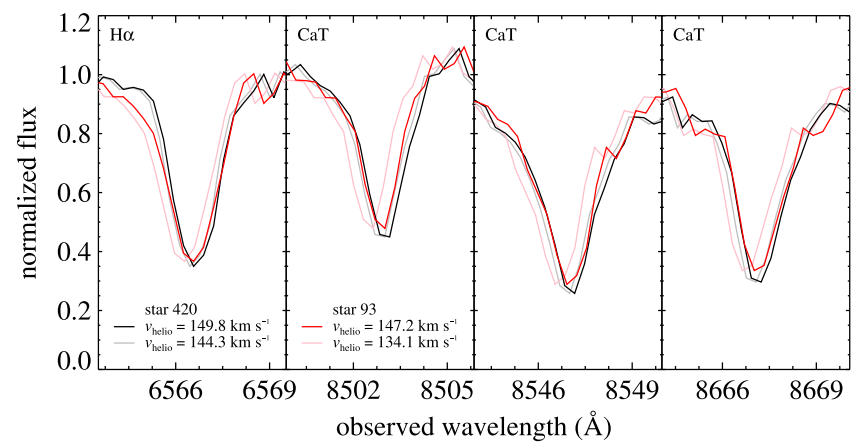

Figure 6. DEIMOS spectra of Laevens 1 stars 420 (black) and 93 (red), which were also observed by Bonifacio et al. (2015). The bold (black and red) spectra show the spectra as we observed them. The faded (gray and pink) spectra show the spectra as they would have appeared at the radial velocities measured by Bonifacio et al. The visible separation between the faded spectra shows that our spectra have the precision to rule out a velocity separation of $10.2 \mathrm{~km} \mathrm{~s}^{-1}$.

Figure 6 shows our DEIMOS spectra in the observer frame. The bold black and red spectra show the spectra as we observed them, and the faded (gray and pink) lines show the spectra as they would have appeared at the velocities measured by Bonifacio et al. (2015). The bold spectra are nearly superposed on top of each other, whereas the faded spectra are visibly separated. Thus, the figure shows that our spectra have the precision to discern between a 10.2 and a $2.6 \mathrm{~km} \mathrm{~s}^{-1}$ difference.

There are several possible reasons for the discrepancy between our two studies. First, one or both of the stars could have a variable radial velocity. In that case, we happened to observe the two stars when they had nearly the same radial velocity, and our inability to resolve the velocity dispersion (Section 5.2) is unaffected. Second, one of our studies could be subject to a systematic error. For example, we corrected for slit 
mis-centering by applying a velocity shift based on telluric absorption. (The wavelength scale in Figure 6 includes the telluric correction.) Bonifacio et al. were unable to apply a similar correction. Therefore, one possible systematic error in their study was slightly different positions of the two stars perpendicular to the X-Shooter slit. Regardless, Bonifacio et al. state that the velocity dispersion of Laevens 1 is consistent with zero when the measurement errors are taken into account. This basic result agrees with our own conclusions (Section 5.2).

We measured $[\mathrm{Fe} / \mathrm{H}]=-1.55 \pm 0.11$ and $-1.65 \pm 0.11$ for stars 420 and 93, respectively. Bonifacio et al. measured $[\mathrm{Fe} / \mathrm{H}]=-1.73 \pm 0.26$ and $-1.67 \pm 0.28$ from an equivalent width (EW) analysis using MyGIsFOS (Sbordone et al. 2014). Our measurements are entirely consistent within the measurement uncertainty.

\section{KINEMATICS}

\subsection{Membership}

Ultra-faint satellites have very low surface brightnesses. In some cases, most of the point sources in the vicinity of an ultrafaint satellite - even at the center-are foreground dwarf stars. We avoided most of the contamination by selecting stars with colors and magnitudes appropriate for red giants at the distance of each satellite (Section 2 and Figure 1). We removed the remainder of the contaminants by imposing a radial velocity cut.

We determined membership in each satellite by using its mean velocity, $\left\langle v_{\text {helio }}\right\rangle$, and velocity dispersion, $\sigma_{v}$. First, we started with guesses for $\left\langle v_{\text {helio }}\right\rangle$ and $\sigma_{v}$. We required member stars to have $\left|v_{\text {helio }}-\left\langle v_{\text {helio }}\right\rangle\right|<2.58 \sigma_{v}$. For a Gaussian velocity distribution, this cut includes $99 \%$ of member stars. Because $\sigma_{v}$ for most of the satellites is very small, a hard cut would exclude low-S/N stars whose measured velocities are discrepant by more than $2.58 \sigma_{v}$ simply because their uncertainties are larger than that. Therefore, we extended the membership criterion to any star whose $1 \sigma$ error bar overlaps the velocity range for member stars. We determined $\left\langle v_{\text {helio }}\right\rangle$ and $\sigma_{v}$ from the list of member stars following the procedure in Section 5.2. Then, we used these new values to reform the member list. We repeated this procedure until the member list did not change from one iteration to the next.

Foreground dwarfs can also be identified by spectral features sensitive to surface gravity, such as the Na I doublet at $8190 \AA$ (Spinrad \& Taylor 1971; Cohen 1978) and Mg I 8807 (Battaglia \& Starkenburg 2012). We found several stars with very strong $\mathrm{Na}$ doublets and $\mathrm{Mg}$ I lines among the Hydra II and Pisces II samples, but they were already excluded by the radial velocity cut. Hence, we did not need to impose any additional membership criteria. We discuss $\mathrm{Mg}$ I again in Section 5.2 in reference to a star that barely missed the membership cut in Hydra II.

Two of the three blue stars that we targeted in Laevens 1 are non-members on the basis of their radial velocities. Belokurov et al. (2014) speculated that these stars might be blue loop stars. If they were, then Laevens 1 must have a young stellar population, which would argue strongly that it is a star-forming galaxy, not a GC. However, the non-membership of these two stars negates most of the evidence that Laevens 1 has a very young stellar population, although Bonifacio et al. (2015) argued that the blue extension of the main sequence might indicate the presence of a $\sim 2$ Gyr old population. The third blue star, 399 , has $v_{\text {helio }}=155.3 \pm 1.8 \mathrm{~km} \mathrm{~s}^{-1}$, which is 6.0 $\mathrm{km} \mathrm{s}^{-1}$ from the mean radial velocity. This star formally passes the velocity membership cut. However, in addition to its unusual color, it has a more discrepant radial velocity than any of the other member stars, and it is farther from the center of the cluster than all but one confirmed member. We considered the possibility that this star is a Cepheid member of Laevens 1. However, the Pan-STARRS1 survey (Kaiser et al. 2010) obtained six observations of this star in each of the $r_{\mathrm{P} 1}, z_{\mathrm{P} 1}$, and $y_{\mathrm{P} 1}$ bands, and the rms scatter of these observations is consistent with measurement noise $(\sim 0.03 \mathrm{mag}$, B. Sesar 2015, private communication). Since Population II Cepheids have light curve amplitudes between 0.5 and $1.2 \mathrm{mag}$ in the Johnson R-band (Wallerstein \& Cox 1984), we conclude that the blue star is not a Cepheid variable. We discuss the impact that including star 399 as a member would have on $\sigma_{v}$ in Section 5.2.

\subsection{Mean Velocities, Velocity Dispersions, and Masses}

We estimated $\left\langle v_{\text {helio }}\right\rangle$ and $\sigma_{v}$ for the three satellites in the same manner that Kirby et al. (2013a) measured these values for the UFD Segue 2. That method, in turn, was based on Walker et al.'s (2006) procedure for measuring the velocity dispersion of the Fornax dwarf spheroidal galaxy (dSph). The method uses maximum likelihood statistics and a Monte Carlo Markov chain (MCMC). We maximized the logarithm of the likelihood $(L)$ that the given values of $\left\langle v_{\text {helio }}\right\rangle$ and $\sigma_{v}$ described the observed velocity distribution, including the uncertainty estimates for individual stars.

$$
\begin{aligned}
\log L= & \frac{N \log (2 \pi)}{2}+\frac{1}{2} \sum_{i}^{N}\left(\log \left(\left(\delta v_{\text {helio }}\right)_{i}^{2}+\sigma_{v}^{2}\right)\right. \\
& +\frac{1}{2} \sum_{i}^{N}\left(\frac{\left(\left(v_{\text {helio }}\right)_{i}-\left\langle v_{\text {helio }}\right\rangle\right)^{2}}{\left(\delta v_{\text {helio }}\right)_{i}^{2}+\sigma_{v}^{2}}\right) .
\end{aligned}
$$

As initial guesses for $\left\langle v_{\text {helio }}\right\rangle$ and $\sigma_{v}$, we started with the mean and standard deviation of $v_{\text {helio }}$ over the velocity range shown in Figure 4. The final results are not sensitive to these guesses. We explored the parameter space with a Metropolis-Hastings implementation of an MCMC. The length of the chain was $10^{7}$ trials.

As a test of our procedure, we measured $\left\langle v_{\text {helio }}\right\rangle=$ $-144.4 \pm 1.3 \mathrm{~km} \mathrm{~s}^{-1}$ and $\sigma_{v}=7.4_{-0.9}^{+1.0} \mathrm{~km} \mathrm{~s}^{-1}$ for M22 from the 2009 October 14 observation. In comparison, Peterson \& Cudworth (1994) measured $\left\langle v_{\text {helio }}\right\rangle=-148.8 \pm 0.8 \mathrm{~km} \mathrm{~s}^{-1}$ and $\sigma_{v}=6.6 \pm 0.8 \mathrm{~km} \mathrm{~s}^{-1}$ within $7^{\prime}$ of the cluster center, and Lane et al. (2009) measured $\left\langle v_{\text {helio }}\right\rangle=-144.9 \pm 0.3 \mathrm{~km} \mathrm{~s}^{-1}$ and a central dispersion of $\sigma_{v}=6.8 \pm 0.9 \mathrm{~km} \mathrm{~s}^{-1}$. Our measurement of $\left\langle v_{\text {helio }}\right\rangle$ is consistent with that of Lane et al. and our measurement of $\sigma_{v}$ is consistent with both Peterson \& Cudworth and Lane et al. The velocity dispersion in M22 decreases to about $5 \mathrm{~km} \mathrm{~s}^{-1}$ at $8^{\prime}$ from the cluster center (Lane et al. 2009). However, our data is largely insensitive to the decline of $\sigma_{v}$ because the maximum radial extent of our spectroscopy is $8^{\prime}$, and most of our M22 targets are within $5^{\prime}$.

Table 3 gives $\left\langle v_{\text {helio }}\right\rangle$ and $\sigma_{v}$ with errors. It also gives velocities relative to the Galactic standard of rest (GSR) assuming that the Sun's orbital velocity is $220 \mathrm{~km} \mathrm{~s}^{-1}$. Hydra II is receding from the Galactic center. Therefore, it is past its pericenter on the way to its apocenter. Its heliocentric radial 
Table 3

Satellite Properties

\begin{tabular}{|c|c|c|c|}
\hline Property & Hydra II & Pisces II & Laevens 1 \\
\hline$N_{\text {member }}$ & 13 & 7 & 10 \\
\hline $\log \left(L_{V} / L_{\odot}\right)$ & $3.90 \pm 0.10$ & $3.93 \pm 0.20$ & $3.65 \pm 0.08$ \\
\hline$r_{\mathrm{h}}(\operatorname{arcmin})$ & $1.7_{-0.2}^{+0.3}$ & $1.1 \pm 0.1$ & $0.47_{-0.03}^{+0.04}$ \\
\hline$r_{\mathrm{h}}(\mathrm{pc})$ & $66_{-9}^{+12}$ & $58 \pm 7$ & $19 \pm 2$ \\
\hline$\left\langle v_{\text {helio }}\right\rangle\left(\mathrm{km} \mathrm{s}^{-1}\right)$ & $303.1 \pm 1.4$ & $-226.5 \pm 2.7$ & $149.3 \pm 1.2$ \\
\hline$v_{\mathrm{GSR}}\left(\mathrm{km} \mathrm{s}^{-1}\right)$ & 135.4 & -79.9 & 4.6 \\
\hline $\log \left(M_{1 / 2} / M_{\odot}\right)$ & $<5.9$ (90\% C.L.) & $6.2_{-0.2}^{+0.3}$ & $<5.5$ (90\% C.L.) \\
\hline$\left(M / L_{V}\right)_{1 / 2}^{\mathrm{a}}\left(M_{\odot} / L_{\odot}\right)$ & $\begin{array}{l}<6.1 \text { (95\% C.L.) } \\
<200 \text { (90\% C.L.) }\end{array}$ & $370_{-240}^{+310}$ & $\begin{array}{l}<5.6 \text { (95\% C.L.) } \\
<130 \text { (90\% C.L.) }\end{array}$ \\
\hline & <315 (95\% C.L.) & & $<192$ (95\% C.L.) \\
\hline$\langle[\mathrm{Fe} / \mathrm{H}]\rangle$ & $-2.02 \pm 0.08$ & $-2.45 \pm 0.07$ & $-1.68 \pm 0.05$ \\
\hline$\sigma([\mathrm{Fe} / \mathrm{H}])$ & $0.40_{-0.26}^{+0.48}$ & $0.48_{-0.29}^{+0.70}$ & $\begin{array}{l}<0.40 \text { (90\% C.L.) } \\
<0.53(95 \% \text { C.L. })\end{array}$ \\
\hline
\end{tabular}

Notes. The measurements of $\log L_{V}$ and $r_{\mathrm{h}}$ come from Martin et al. (2015), Belokurov et al. (2010), and Laevens et al. (2014).

${ }^{a}$ Mass-to-light ratio within the half-light radius.

velocity is also similar to that of the gas in the leading arm of the Magellanic stream (Putman et al. 1998; Brüns et al. 2005; Nidever et al. 2008). This finding strengthens the potential for Hydra II to be associated with the LMC (Bechtol et al. 2015; Deason et al. 2015; Koposov et al. 2015a). Pisces II is approaching the Galactic center, which means that it is on the way to its pericenter. Finally, Laevens 1 has a very small $v_{\mathrm{GSR}}$, meaning that it is at pericenter or apocenter. Because it is so distant (at least $140 \mathrm{kpc}$ from the Galactic center), it is either close to apocenter or on a quasi-circular orbit.

Figure 7 shows the distribution of $\sigma_{v}$ for accepted MCMC trials. These distributions are equivalent to the probability distribution for $\sigma_{v}$. The distribution for Pisces II has a welldefined peak separated from zero. The dashed line shows the median value of $\sigma_{v}=5.4 \mathrm{~km} \mathrm{~s}^{-1}$. We estimated asymmetric error bars by calculating the values of $\sigma_{v}$ that bracket $68.3 \%$ of the probability distribution.

The distributions for Hydra II and Laevens 1 are concentrated near zero. Hence, our measurements cannot resolve $\sigma_{v}$ for these two satellites. We estimated two sets of upper limits by finding the value of $\sigma_{v}$ that exceeds $90 \%$ and $95 \%$ of the MCMC trials. Table 3 gives both upper limits for both satellites.

Hydra II and Laevens 1 have three stars with somewhat ambiguous membership. Stars 197129 and 188838 in Hydra II have velocities larger than $\left\langle v_{\text {helio }}\right\rangle$ by 12.6 and $15.9 \mathrm{~km} \mathrm{~s}^{-1}$. If our membership cut were more inclusive than $2.58 \sigma_{v}$, then these stars could be considered members. Unfortunately, neither star has a visible $\mathrm{Na}$ I doublet to help define its status. The $\mathrm{Mg}$ I EW of star 188838 is $0.34 \pm 0.07 \AA$, and the combined EW of of the two redder lines of the $\mathrm{Ca}$ II infrared triplet $(\mathrm{CaT})$ is $4.1 \pm 0.3 \AA$. These measurements fall right on the dividing line between dwarfs and giants defined by Battaglia \& Starkenburg (2012). Because most metal-poor $\mathrm{dSph}$ stars fall well below the dividing line, this test disfavors membership for star 188838. The $\mathrm{Mg}_{\mathrm{I}}$ line in star 197129 is too noisy to be useful.

Including star 192179 as a member does not change our qualitative conclusions. Instead, the $90 \%$ confidence level

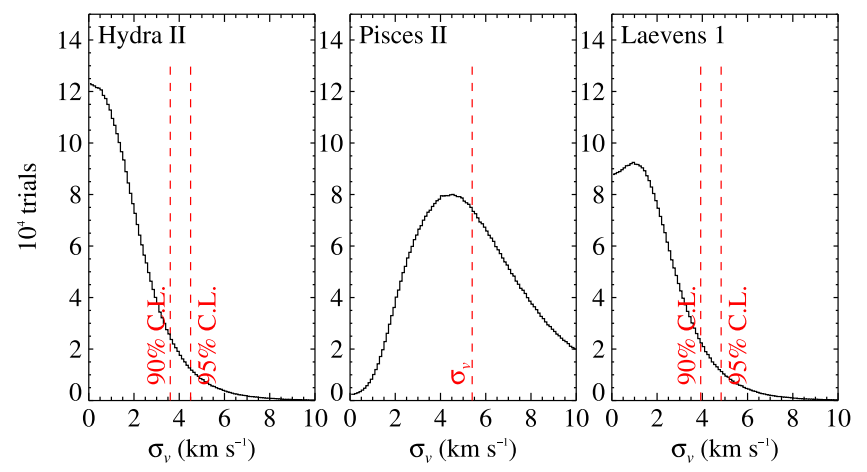

Figure 7. Probability distributions for $\sigma_{v}$. The histograms show successful MCMC trials. Vertical lines show the measured velocity dispersion (Pisces II) or upper limits (Hydra II and Laevens 1).

(C.L.) upper limit on $\sigma_{v}$ rises from 3.6 to $6.0 \mathrm{~km} \mathrm{~s}^{-1}$. On the other hand, including star 188838 resolves the velocity dispersion as $\sigma_{v}=6.4_{-1.8}^{+2.4} \mathrm{~km} \mathrm{~s}^{-1}$. Including both stars results in $\sigma_{v}=6.7_{-1.9}^{+2.4} \mathrm{~km} \mathrm{~s}^{-1}$. However, both stars would be $>2 \sigma_{v}$ outliers even with the larger $\sigma_{v}$. Furthermore, Figure 1 shows that both stars are redder relative to the M92 isochrone than any other member. Figure 2 also shows that star 188838 would be the most distant member of Hydra II. Because the majority of the evidence disfavors membership, we ruled both stars as nonmembers. Including or excluding the two possible HB stars, 189086 and 191385 , changes the limits on $\sigma_{v}$ by less than $2 \%$. Based on their CMD positions, we ruled 189086 a member and 191385 a non-member.

Including star 399 in Laevens 1 resolves the velocity dispersion as $\sigma_{v}=2.7_{-1.4}^{+1.8} \mathrm{~km} \mathrm{~s}^{-1}$. As discussed in Section 5.1, the star is bright and blue. As a result, star 399 is probably not a member.

The total mass of a spherical stellar system in dynamical equilibrium is related to the square of the velocity dispersion. Wolf et al. (2010) showed that the total mass is poorly constrained because any possible underlying dark matter has an 
unknown mass profile, and the velocity anisotropy cannot be determined from a small sample of radial velocities. However, the mass within the half-light radius, $M_{1 / 2}$, is well-constrained: $M_{1 / 2}=4 G^{-1} \sigma_{v}^{2} r_{\mathrm{h}}$. Although $r_{\mathrm{h}}$ is the two-dimensional (2D) half-light radius, the formula infers the mass enclosed within the three-dimensional (3D) half-light radius.

Table 3 gives $\log M_{1 / 2}$ for Pisces II and upper limits for Hydra II and Laevens 1, which have only upper limits for $\sigma_{v}$. Pisces II has a dynamical mass on par with dwarf galaxies of similar luminosity (Strigari et al. 2008; Wolf et al. 2010). For example, its luminosity and mass are very similar to Canes Venatici II (Simon \& Geha 2007). The mass limits for Hydra II and Laevens 1 are not stringent enough to make these satellites unusually light. The 90\% C.L. for Hydra II is $M_{1 / 2}<1.0 \times 10^{6} M_{\odot}$, which is less than a factor of 2 smaller than Pisces II. The $90 \%$ C.L. mass limit for Segue 2, the least massive galaxy, is 7 times more stringent than Hydra II. The limit for Laevens 1 is slightly more stringent than Hydra II, but we argue below that Laevens 1 is not a galaxy.

Willman \& Strader (2012) proposed that a "galaxy" should be defined as a stellar system that cannot be explained by a combination of baryons and Newton's laws of gravity. The $M / L$ for Pisces II is $370_{-240}^{+310} M_{\odot} / L_{\odot}$. This value is far too large to be explained by baryons alone, even for the oldest stellar populations in the universe. In fact, $99 \%$ of the successful MCMC trials have $M / L>10 M_{\odot} / L_{\odot}$. Hence, Pisces II satisfies the definition of a galaxy. However, upper limits on the $M / L s$ on Hydra II and Laevens 1 do not help in deciding if they are galaxies. In Section 6, we use chemical evidence to resolve the nature of these two satellites.

One of the reasons dwarf galaxies are interesting is that they are targets for the detection of gamma rays due to dark matter self-annihilation. Pisces II has similar structural properties $\left(r_{\mathrm{h}}\right.$ and $\sigma_{v}$ ) and distance to Canes Venatici II. Hence, the two galaxies' potential for the detection of the gamma-ray signal is about the same. Bonnivard et al. (2015) found that Canes Venatici II is not the most promising dwarf galaxy to search for self-annihilation, but it would contribute significantly to an analysis that stacks the Fermi gamma-ray telescope observations of all of the dwarfs (e.g., Ackermann et al. 2014, 2015).

\section{METALLICITY}

The stellar mass of a UFD is insufficient to retain supernova ejecta. Nonetheless, all UFDs studied in sufficient detail show evidence for metallicity dispersions (e.g., Frebel et al. 2010; Norris et al. 2010; Kirby et al. 2013a; Vargas et al. 2013). Their ability to self-enrich with iron implies that they have or once had enough mass to prevent metal-enriched supernova ejecta from escaping. As a result, Willman \& Strader (2012) considered a dispersion in $[\mathrm{Fe} / \mathrm{H}]$ sufficient for classification as a galaxy for most stellar systems. ${ }^{8}$

We calculated the metallicity mean and dispersion in the same way that we computed $\left\langle v_{\text {helio }}\right\rangle$ and $\sigma_{v}$. In analogy to Equation (1), we maximized the likelihood that the metallicity distribution has a mean $\langle[\mathrm{Fe} / \mathrm{H}]\rangle$ and dispersion $\sigma([\mathrm{Fe} / \mathrm{H}])$ as

\footnotetext{
8 Some massive GCs, like $\omega$ Centauri (Norris \& Da Costa 1995), have a dispersion in metallicity but no kinematic evidence for dark matter.
}

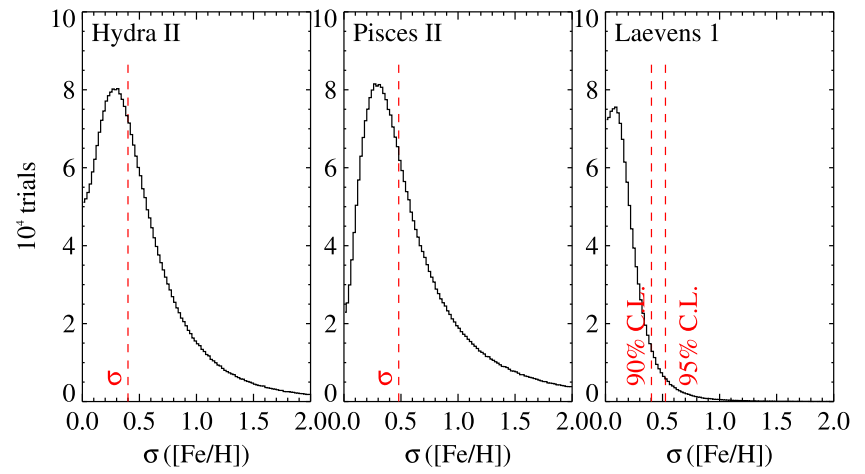

Figure 8. Probability distributions for $\sigma([\mathrm{Fe} / \mathrm{H}])$. The histograms show successful MCMC trials. Vertical lines show the measured metallicity dispersions (Hydra II and Pisces II) or upper limits (Laevens 1).

follows:

$$
\begin{aligned}
\log L= & \frac{N \log (2 \pi)}{2} \\
& +\frac{1}{2} \sum_{i}^{N}\left(\log (\delta[\mathrm{Fe} / \mathrm{H}])_{i}^{2}+\sigma([\mathrm{Fe} / \mathrm{H}])^{2}\right) \\
& +\frac{1}{2} \sum_{i}^{N}\left(\frac{\left([\mathrm{Fe} / \mathrm{H}]_{i}-\langle[\mathrm{Fe} / \mathrm{H}]\rangle\right)^{2}}{(\delta[\mathrm{Fe} / \mathrm{H}])_{i}^{2}+\sigma([\mathrm{Fe} / \mathrm{H}])^{2}}\right)
\end{aligned}
$$

We used the metallicity measurements shown in Table 2, i.e., those with $\delta[\mathrm{Fe} / \mathrm{H}]<0.5$ and $\mathrm{S} / \mathrm{N}>20 \AA^{-1}$. The values were determined through $10^{7}$ MCMC trials.

Figure 8 shows the distribution of $\sigma([\mathrm{Fe} / \mathrm{H}])$ for the successful MCMC trials. Whereas the distributions for Hydra II and Pisces II are somewhat separated from zero, the distribution for Laevens 1 piles up at zero. Hence, we marginally resolve the metallicity dispersion for Hydra II and Pisces II: $79 \%$ and $85 \%$ of the MCMC trials have $\sigma_{[\mathrm{Fe} / \mathrm{H}]}>0.2$, respectively. On the other hand, we measured only an upper limit for Laevens 1 . These values are shown in Table 3.

Although we did not resolve the velocity dispersion for Hydra II, we did detect a metallicity dispersion, albeit with a somewhat low significance. Because it seems that Hydra II enriched itself with iron, we tentatively assign it a classification as a galaxy. This spectroscopic classification supports the photometric classification based on its large half-light radius (Martin et al. 2015). Pisces II also has a marginally resolved metallicity dispersion, consistent with its dynamical classification as a galaxy.

Dwarf galaxies obey a tight relationship between luminosity and average metallicity (Skillman et al. 1989; Mateo 1998; Kirby 2011; Kirby et al. 2013b). In fact, the luminositymetallicity relation (LZR) can be used as a diagnostic for whether a stellar system is a galaxy. Figure 9 shows the LZR from Kirby et al. (2013b) with the three new satellites. Hydra II and Pisces II lie in the same region of the diagram as other UFDs, like Leo IV and Canes Venatici II. Thus, the LZR supports their identification as galaxies.

On the other hand, Laevens 1 is too metal-rich for its luminosity (or too faint for its metallicity). GCs do not obey any LZR. Tidal dwarf galaxies also do not obey the LZR, but tidal dwarfs tend to be close to solar metallicity (Duc et al. 2000). Therefore, Laevens 1's position in Figure 9 suggests that it is a GC or a severely tidally stripped dwarf 


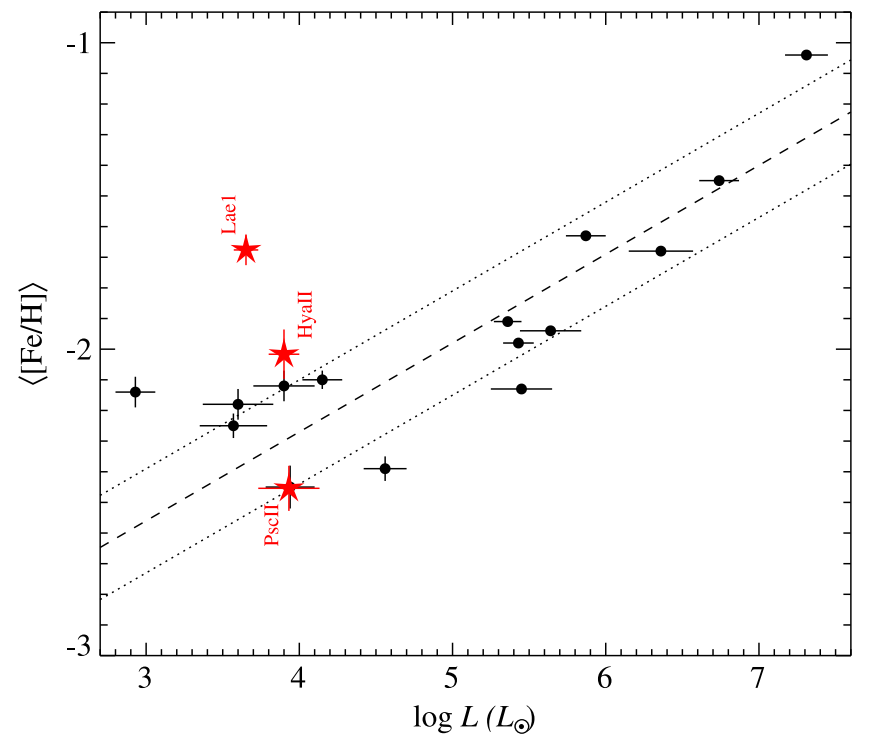

Figure 9. Luminosity-metallicity relation for Milky Way satellite galaxies. Hydra II and Pisces II fall in the midst of other UFDs. Pisces II falls almost directly on top of Leo IV. Like other GCs, Laevens 1 does not conform to the relation. The black points are $\langle[\mathrm{Fe} / \mathrm{H}]\rangle$ measurements for Milky Way dSphs from Kirby et al. (2013b). The dashed line is a least-squares fit to the black points excluding Segue 2, the least luminous galaxy on the plot. The dotted lines show the rms dispersion about the relation.

galaxy, in which the removal of stars has reduced Laevens 1's mass but not its metallicity. Kirby et al. (2013a) proposed this scenario for Segue 2, but Segue 2 has a metallicity dispersion. We detected no metallicity dispersion in Laevens 1, favoring its identification as a GC.

Laevens 1 has four pieces of evidence that suggest that it is a GC, not a galaxy. First, it has a smaller half-light radius than known UFDs (Laevens et al. 2014). Second, it does not have strong evidence for dark matter $\left(M / L<130 M_{\odot} / L_{\odot}\right.$, $90 \%$ C.L.). Third, its metallicity dispersion is less than 0.40 dex $(90 \%$ C.L.). Fourth, it does not obey the LZR for dwarf galaxies. Additionally, most of the blue stars that may have suggested that Laevens 1 has a young stellar population turned out to be probable non-members (Section 5.1). The various lines of evidence taken together favor a cluster classification rather than a dwarf galaxy.

Star 10694, the brightest member of Pisces II, is carbon-rich. Figure 3 shows CN absorption between 8300 and $8400 \AA$. Bright red giants should be destroying carbon, not creating it. Star 10694 could be an AGB star that is currently dredging up carbon. Alternatively, it could have acquired carbon from a recently defunct AGB companion. Its large luminosity favors the scenario that it is itself an AGB star.

\section{SUMMARY}

We obtained Keck/DEIMOS spectroscopy for three newly discovered Milky Way satellites: Hydra II, Pisces II, and Laevens 1. We measured radial velocities and metallicities for individual candidate member stars. We identified 13, 7, and 10 member stars in the three satellites, respectively, on the basis of their radial velocities. Most of the members are red giants, although there is one HB and one AGB star in Hydra II and one carbon-rich star in Pisces II.

We could not resolve the velocity dispersion of Hydra II, but we did measure a non-zero dispersion in metallicity
$\left(\sigma([\mathrm{Fe} / \mathrm{H}])=0.40_{-0.26}^{+0.48}\right)$. Because it seems to have chemically enriched itself in iron and because it has a large half-light radius (66 pc, Belokurov et al. 2010), Hydra II is more likely to be a dwarf galaxy than a GC. It also has a radial velocity similar to the gas in the leading arm of the Magellanic stream at its location. Therefore, it may have fallen into the Milky Way with the Magellanic Clouds.

Pisces II is a bona fide galaxy that inhabits a massive dark matter halo. We measured a velocity dispersion in Pisces II that is far in excess of what would be expected on the basis of its stellar mass alone. We also marginally resolved a metallicity dispersion $\left(\sigma([\mathrm{Fe} / \mathrm{H}])=0.48_{-0.29}^{+0.70}\right)$, indicating that Pisces II is not only massive now, but it was also massive enough during star formation to retain supernova ejecta.

Laevens 1 is more likely a GC than a galaxy. We did not resolve a dispersion in velocity or metallicity, and Laevens 1 does not obey the LZR for dwarf galaxies, whereas Hydra II and Pisces II do. Although the upper limit on the $M / L$ $\left(M / L<130 M_{\odot} / L_{\odot}, 90 \%\right.$ C.L. $)$ does not rule out that Laevens 1 could be dark matter-dominated, the low metallicity dispersion and the inconsistency with the LZR suggest that the system is a GC.

Belokurov et al. (2014) found several bright, blue stars in the vicinity of Laevens 1 . They suggested that these could be blue loop stars, which would signify the presence of a very young stellar population in Laevens 1 . However, we found these stars to be probable non-members on the basis of radial velocity.

With this work, we have spectroscopically confirmed two dwarf galaxies and one GC. The dwarf galaxies are especially interesting for their application to dark matter physics. In particular, Pisces II has a $M / L$ within the half-light radius of $370_{-240}^{+310} M_{\odot} / L_{\odot}$. Although this does not make it one of the most dark matter-dominated galaxies known, it is still massive enough to warrant inclusion in a search for gamma rays due to dark matter self-annihilation (e.g., Ackermann et al. 2014, 2015; Bonnivard et al. 2015).

We thank D. Perley for obtaining LRIS images of Laevens 1, J.A. Newman for assistance with the DEIMOS wavelength solution, E. Tollerud for the spectrum of the radial velocity template star HD 38230, and M. de los Reyes for pointing out that tidal dwarf galaxies do not obey the LZR. We also thank B. Sesar and the Pan-STARRS team for information regarding star 399 in Laevens 1 . The referee's helpful comments significantly improved this article. We are grateful to the many people who have worked to make the Keck Telescope and its instruments a reality and to operate and maintain the Keck Observatory. The authors wish to extend special thanks to those of Hawaiian ancestry on whose sacred mountain we are privileged to be guests. Without their generous hospitality, none of the observations presented herein would have been possible.

Facility: Keck:I (LRIS), Keck:II (DEIMOS).

\section{REFERENCES}

Abazajian, K. N., Adelman-McCarthy, J. K., Agüeros, M. A., et al. 2009, ApJS, 182, 543

Ackermann, A., Albert, A., Anderson, B., et al. 2015, PhRvD, 19, 122002

Ackermann, M., Albert, A., Anderson, B., et al. 2014, PhRvD, 89, 042001

Anders, E., \& Grevesse, N. 1989, GeCoA, 53, 197

Battaglia, G., \& Starkenburg, E. 2012, A\&A, 539, A123

Bechtol, K., Drlica-Wagner, A., Balbinot, E., et al. 2015, ApJ, 807, 50

Belokurov, V., Irwin, M. J., Koposov, S. E., et al. 2014, MNRAS, 441, 2124

Belokurov, V., Walker, M. G., Evans, N. W., et al. 2010, ApJL, 712, L103 
Belokurov, V., Zucker, D. B., Evans, N. W., et al. 2007, ApJ, 654, 897

Bertin, E., \& Arnouts, S. 1996, A\&AS, 117, 393

Bonifacio, P., Caffau, E., Zaggia, S., et al. 2015, A\&A, 579, L6

Bonnivard, V., Combet, C., Daniel, M., et al. 2015, MNRAS, submitted (arXiv:1504.02048)

Brüns, C., Kerp, J., Staveley-Smith, L., et al. 2005, A\&A, 432, 45

Clem, J. L. 2006, PhD thesis, Univ. Victoria

Cohen, J. G. 1978, ApJ, 221, 788

Cooper, M. C., Newman, J. A., Davis, M., Finkbeiner, D. P., \& Gerke, B. F. 2012, ascl soft, ascl:1203.003

Deason, A. J., Wetzel, A. R., Garrison-Kimmel, S., \& Belokurov, V. 2015, ApJL, submitted (arXiv:1504.04372)

Duc, P.-A., Brinks, E., Springel, V., et al. 2000, AJ, 120, 1238

Faber, S. M., Phillips, A. C., Kibrick, R. I., et al. 2003, Proc. SPIE, 4841, 1657

Frebel, A., Simon, J. D., Geha, M., \& Willman, B. 2010, ApJ, 708, 560

Frebel, A., Simon, J. D., \& Kirby, E. N. 2014, ApJ, 786, 74

Harris, W. E. 1996, AJ, 112, 1487

Jordi, K., Grebel, E. K., \& Ammon, K. 2006, A\&A, 460, 339

Kaiser, N., Burgett, W., Chambers, K., et al. 2010, Proc. SPIE, 7733

Kim, D., \& Jerjen, H. 2015a, ApJ, 799, 73

Kim, D., \& Jerjen, H. 2015b, ApJL, 808, L39

Kim, D., Jerjen, H., Mackey, D., Da Costa, G. S., \& Milone, A. P. 2015a, ApJL, 804, L44

Kim, D., Jerjen, H., Milone, A. P., Mackey, D., \& Da Costa, G. S. 2015b, ApJ, 803,63

Kirby, E. N. 2011, PASP, 123, 531

Kirby, E. N., Boylan-Kolchin, M., Cohen, J. G., et al. 2013a, ApJ, 770, 16

Kirby, E. N., Cohen, J. G., Guhathakurta, P., et al. 2013b, ApJ, 779, 102

Kirby, E. N., Guhathakurta, P., Simon, J. D., et al. 2010, ApJS, 191, 352

Kirby, E. N., Guhathakurta, P., \& Sneden, C. 2008a, ApJ, 682, 1217

Kirby, E. N., Guo, M., Zhang, A. J., et al. 2015, ApJ, 801, 125

Kirby, E. N., Simon, J. D., Geha, M., Guhathakurta, P., \& Frebel, A. 2008b, ApJL, 685, L43

Koposov, S. E., Belokurov, V., Torrealba, G., \& Evans, N. W. 2015a, ApJ, 805,130

Koposov, S. E., Casey, A. R., Belokurov, V., et al. 2015b, ApJ, submitted (arXiv:1504.07916)

Kurucz, R. 1993, ATLAS9 Stellar Atmosphere Programs and $2 \mathrm{~km} \mathrm{~s}^{-1}$ grid. Kurucz CD-ROM No. 13 (Cambridge, MA: Smithsonian Astrophysical Observatory), 13
Laevens, B. P. M., Martin, N. F., Ibata, R. A., et al. 2015, ApJL, 802, L18

Laevens, B. P. M., Martin, N. F., Sesar, B., et al. 2014, ApJL, 786, L3

Lane, R. R., Kiss, L. L., Lewis, G. F., et al. 2009, MNRAS, 400, 917

Martin, N. F., Nidever, D. L., Besla, G., et al. 2015, ApJL, 804, L5

Mateo, M. L. 1998, ARA\&A, 36, 435

Newman, J. A., Cooper, M. C., Davis, M., et al. 2013, ApJS, 208, 5

Nidever, D. L., Bovy, J., Bird, J. C., et al. 2015, American Astronomical Society Meeting Abstracts, 225, 319.01

Nidever, D. L., Majewski, S. R., \& Burton, W. B. 2008, ApJ, 679, 432

Norris, J. E., \& Da Costa, G. S. 1995, ApJ, 447, 680

Norris, J. E., Wyse, R. F. G., Gilmore, G., et al. 2010, ApJ, 723, 1632

Oke, J. B., Cohen, J. G., Carr, M., et al. 1995, PASP, 107, 375

Peterson, R. C., \& Cudworth, K. M. 1994, ApJ, 420, 612

Putman, M. E., Gibson, B. K., Staveley-Smith, L., et al. 1998, Natur, 394,752

Sand, D. J., Strader, J., Willman, B., et al. 2012, ApJ, 756, 79

Sbordone, L., Caffau, E., Bonifacio, P., \& Duffau, S. 2014, A\&A, 564, A109

Schlegel, D. J., Finkbeiner, D. P., \& Davis, M. 1998, ApJ, 500, 525

Shanks, T., Metcalfe, N., Chehade, B., et al. 2015, MNRAS, 451, 4238

Simon, J. D., Drlica-Wagner, A., Li, T. S., et al. 2015, ApJ, 808, 95

Simon, J. D., \& Geha, M. 2007, ApJ, 670, 313

Simon, J. D., Geha, M., Minor, Q. E., et al. 2011, ApJ, 733, 46

Skillman, E. D., Kennicutt, R. C., \& Hodge, P. W. 1989, ApJ, 347, 875

Sneden, C., Kraft, R. P., Prosser, C. F., \& Langer, G. E. 1992, AJ, 104, 2121

Sneden, C. A. 1973, PhD thesis, Univ Texas

Spinrad, H., \& Taylor, B. J. 1971, ApJS, 22, 445

Stetson, P. B. 1987, PASP, 99, 191

Stetson, P. B. 2011, ascl soft, ascl:1104.011

Strigari, L. E., Bullock, J. S., Kaplinghat, M., et al. 2008, Natur, 454, 1096 Tonry, J., \& Davis, M. 1979, AJ, 84, 1511

Vargas, L. C., Geha, M., Kirby, E. N., \& Simon, J. D. 2013, ApJ, 767, 134

Walker, M. G., Mateo, M., Olszewski, E. W., et al. 2006, AJ, 131, 2114

Walker, M. G., Mateo, M., Olszewski, E. W., et al. 2015, ApJ, 808, 108

Wallerstein, G., \& Cox, A. N. 1984, PASP, 96, 677

Willman, B., Blanton, M. R., West, A. A., et al. 2005, AJ, 129, 2692

Willman, B., \& Strader, J. 2012, AJ, 144, 76

Wolf, J., Martinez, G. D., Bullock, J. S., et al. 2010, MNRAS, 406, 1220

Yanny, B., Rockosi, C., Newberg, H. J., et al. 2009, AJ, 137, 4377

Zucker, D. B., Belokurov, V., Evans, N. W., et al. 2006, ApJL, 650, L41 